(C) Elsevier Sequoia S.A., Lausanne - Printed in Switzerland

\title{
TEMPERATURE AND SOLVENT EFFECTS ON PHOTOLUMINESCENCE OF TRYPTOPHAN SOLUTIONS
}

\author{
L. C. PEReira, I. C. FERREira, MARILIA P. F. THOMAZ ${ }^{+}$and M. ISABel B. $M$. \\ JORGE $^{\ddagger}$ \\ University of Minho, Molecular Physics Group, Braga (Portugal)
}

(Received January 27, 1978)

\section{Summary}

The fluorescence of tryptophan solutions in glycerol, water and ethanol was measured as a function of temperature using steady state and timeresolved techniques. The non-radiative rate constants responsible for the variation of fluorescence quantum yields with temperature are evaluated. Spectral shifts occurring on the nanosecond scale are interpreted in terms of an inversion of ${ }^{1} L_{b}$ and ${ }^{1} L_{a}$ states produced by solvent perturbation.

\section{Introduction}

The effects of the environment on the excited states of tryptophan solutions is a subject of considerable interest since luminescence of proteins arises mostly from tryptophan residues. The knowledge of the excited state parameters in different media can be used to predict the behaviour of protein properties [1 - 3]. The effects of polar solvents on the fluorescence of indoles and tryptophan have been investigated by many authors [4] using steady state measurements and fluorescence lifetimes but time-resolved fluorescence has not been extensively used.

A study of the fluorescence of dilute solutions of tryptophan in ethanol, glycerol and water at various temperatures using both steady state and timeresolved fluorescence techniques is reported here. Time-resolved luminescence in the nanosecond and subnanosecond regions is a promising technique in the field of physical biochemistry. Some examples of ways in which this technique may make a large contribution are investigations of possible fluctuations in protein structures [5] and of solvent-lattice interactions with excited states existing for very short times.

\footnotetext{
${ }^{\dagger}$ Present address: University of Aveiro, Physics Department, Portugal.

¥ Present address: University of Mondlane, Physics Department, Maputo, Mozambique.
} 


\section{Experimental}

\subsection{Materials}

L-Tryptophan and the solvents used were of high purity grade. Ethanol and glycerol were purified further by standard methods. Water was deionized and triply distilled. No impurities were detected in any of the solvents by luminescence and absorption techniques.

\subsection{Absorption spectra}

Absorption spectra were recorded in a Pye Unicam SP1800 spectrometer.

\subsection{Fluorescence spectra}

Fluorescence spectra were measured using steady state illumination on a spectrofluorimeter described elsewhere [6] and on a Perkin Elmer MPF44A spectrometer with the spectral correction determined by using a calibrated tungsten lamp and suitable quantum counters [7]. A concentration of $10^{-4} \mathrm{M}$ was used in most of the samples. Fluorescence quantum yields were determined at room temperature and as a function of temperature using samples in an argon atmosphere or in vacuum. Details of the method and precautions used have been described elsewhere [8]. Polarization effects introduced by the apparatus and by fluorescence polarization were investigated in the wavelength and temperature ranges under consideration in order to minimize errors due to anisotropic photoselection [9]. The excitation wavelength was kept at $280 \mathrm{~nm}$ and the exciting light was cut off between measurements to avoid photochemical reactions [10].

\subsection{Time-resolved fluorescence}

Time-resolved fluorescence spectra were obtained using a conventional pulse fluorimeter with a free-running high pressure nitrogen flash lamp. Details of the apparatus have been described by Thomaz et al. [11].

\section{Results and discussion}

Fluorescence spectra show a pronounced wavelength shift in different media which has no counterpart in absorption. This effect is illustrated in Fig. 1 for microcrystalline powder samples and for solutions in ethanol, glycerol and water at room temperature in which the corrected spectra were normalized to the maximum intensity. Spectral shifts were also observed for the same solvents as a function of temperature. This effect is shown for glycerol solutions below room temperature in Fig. 2. In contrast to the results obtained in ethanol, where the fluorescence only showed a small shift below $173 \mathrm{~K}$, the fluorescence of tryptophan in glycerol shows a marked wavelength shift below room temperature. A variation of the maximum wavelength $\lambda_{\max }$ from $324 \pm 1 \mathrm{~nm}$ at $173 \mathrm{~K}$ to $341 \pm 1 \mathrm{~nm}$ at room temperature $(295 \mathrm{~K})$ is observed in this case. The results obtained for the variation of $\lambda_{\max }$ as a func- 


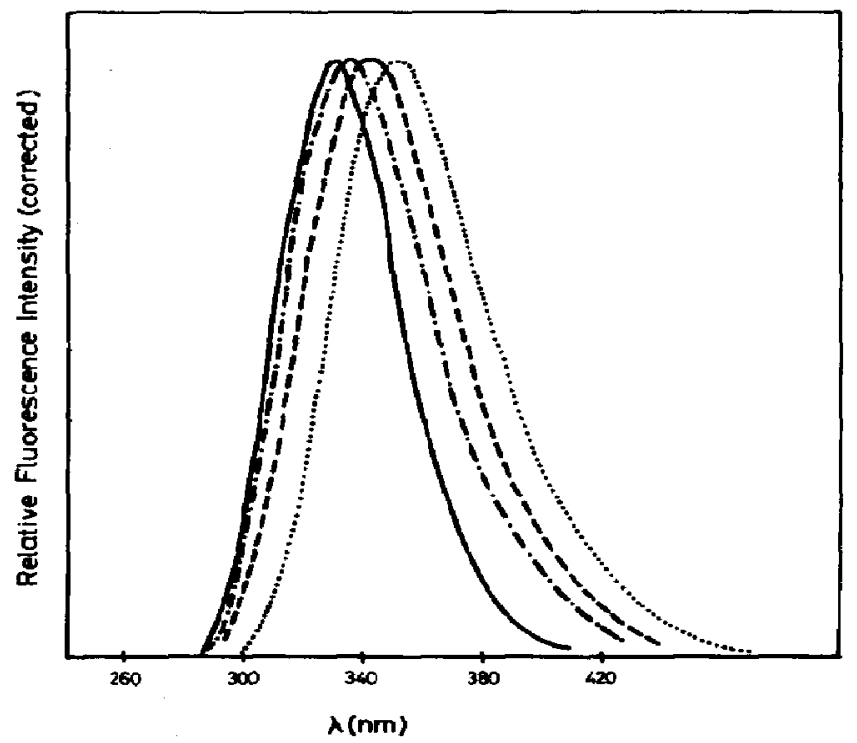

Fig. 1. Fluorescence spectra of tryptophan at room temperature normalized to the maximum intensity : microcrystalline powder; -.- ethanol ; glycerol; water.

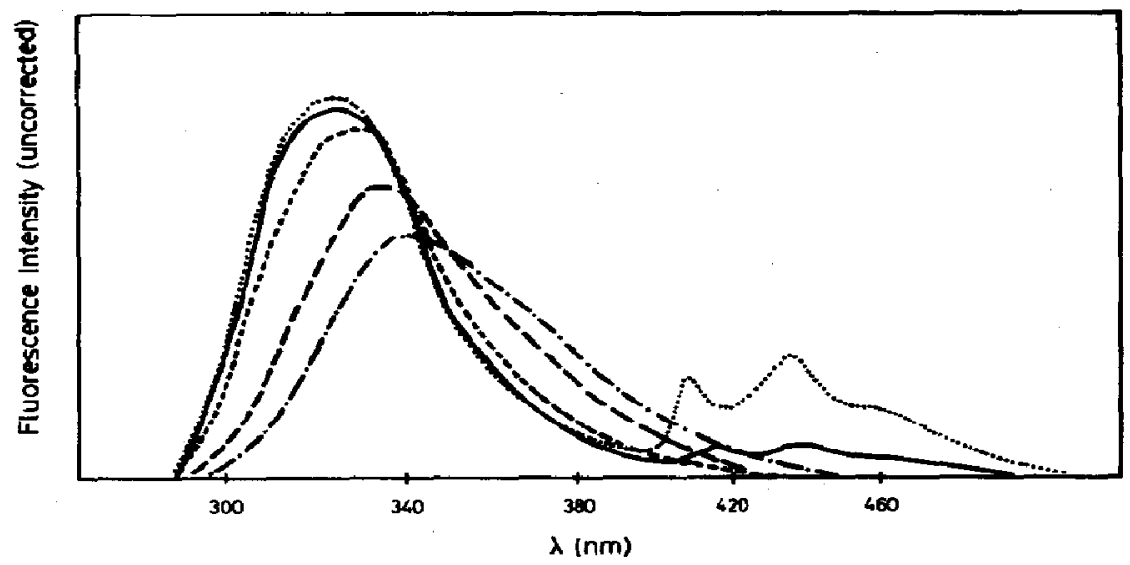

Fig. 2. Emission spectra of tryptophan in glycerol at various temperatures (in Kelvins): $\ldots . ., 173 ;-203 ; . . .-., 233 ;--, 261 ;-.-, 300$.

tion of temperature and solvent are illustrated in Fig. 3. At lower temperatures the fluorescence spectra do not shift and phosphorescence is also observable for glycerol solutions.

A similar effect on the shift of fluorescence spectra with temperature was reported by Eisinger and Navon [12] for tryptophan dissolved in a mixture of ethylene glycol and water (EGW).

Fluorescence quantum yields $\phi_{\mathrm{FM}}$ were determined in water, ethanol and glycerol as a function of temperature. For neutral aqueous solutions $\phi_{\text {FM }}$ was determined between room temperature and $359 \mathrm{~K}$. For solutions in 


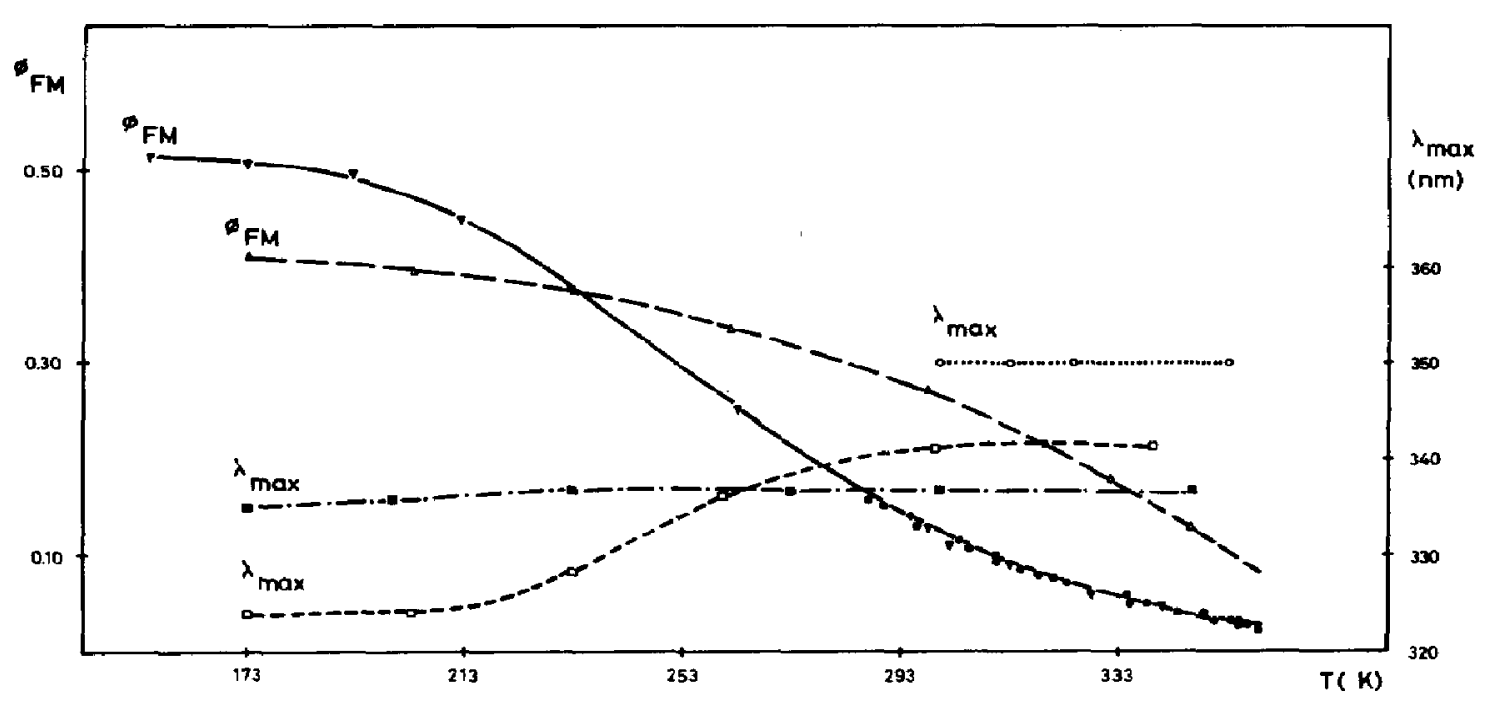

Fig. 3. Variation of $\lambda_{\max }$ of fluorescence and of $\phi_{\mathrm{FM}}$ as functions of solvent and temperature. Fluorescence yields in ethanol $(\nabla)$, glycerol $(\Delta)$ and water $(\bullet) ; \lambda_{\max }$ in ethanol $(*)$, glycerol (a) and water (o)

ethanol and glycerol $\phi_{\mathrm{FM}}$ was determined both above and below room temperature, down to 173 and $155 \mathrm{~K}$ respectively in glycerol and ethanol. The results obtained are also shown in Fig. 3. Some discrepancies are found in the literature for $\phi_{\mathrm{FM}}$ values of tryptophan. For example, while Eisinger and Navon [12] reported $\phi_{\mathrm{FM}}=0.14$ in aqueous solutions at $300 \mathrm{~K}$, Tatischeff and Klein [10] found $\phi_{\mathrm{FM}}=0.13$ in water at room temperature and Teale and Weber [13] found $\phi_{\mathrm{FM}}=0.20$. We have assumed a value of 0.13 for the fluorescence quantum yield of aqueous solutions at $298 \mathrm{~K}$ and have determined the fluorescence quantum yields in glycerol and ethanol relative to water at the same temperature.

Fluorescence quantum yields in ethanol reach a value of 0.51 which is consistent with the results obtained by Eisinger and Navon [12] for EGW solutions. The variation with temperature of the $\phi_{\mathrm{FM}}$ of tryptophan in glycerol is less pronounced and a plateau value is not reached at $173 \mathrm{~K}$. It must be pointed out that if an exciplex is formed in glycerol solutions the total intensity cannot be assigned to $\phi_{\mathrm{FM}}$.

The results seem to indicate that the total fluorescence intensity tends to an approximately constant value at lower temperatures. Assuming that $k_{\mathrm{FM}}=k_{\mathrm{FM}}^{\mathbf{0}} n^{2}$, as found by Cundall and Pereira [14] for benzenoid molecules in various solvents, and using the values of $\phi_{\mathrm{FM}}$ and the fluorescence lifetimes $\tau_{M}$ determined by Eisinger and Navon [12] it is possible to estimate the fluorescence rate constants $k_{\mathrm{FM}}$ for the different solvents. It is reasonable to assume that the non-radiative rate constant $k_{\mathrm{i}}$ is of the type

$$
k_{\mathrm{i}}=k_{\mathrm{i}}^{0}+A \exp (-\Delta E / k T)
$$

and that in the lower temperature region lim $k_{1} \rightarrow k_{i}^{0}$. Therefore, by plotting $\log \left\{\left(1 / \phi_{\mathrm{FM}}-1\right) k_{\mathrm{FM}}-k_{\mathrm{i}}^{\mathrm{o}}\right\}$ versus $1 / T$ the following values of $k_{\mathrm{i}}^{\mathrm{o}}, A$ and $\Delta E$ 
are obtained: glycerol solutions, $k_{\mathrm{i}}^{0}=8.0 \times 10^{7} \mathrm{~s}^{-1}, A=1.3 \times 10^{10} \mathrm{~s}^{-1}$ and $\Delta E=0.13 \mathrm{eV} ;$ ethanol solutions, $k_{i}^{0}=5.3 \times 10^{7} \mathrm{~s}^{-1}, A=8.1 \times 10^{11} \mathrm{~s}^{-1}$ and $\Delta E=0.20 \mathrm{eV}$. In the range of temperatures investigated the results for $\phi_{\mathrm{FM}}$ in water are similar to those obtained in ethanol. Arrhenius plots show a good linear fit for water and ethanol solutions but for glycerol the linear region is somewhat uncertain.

The results for the variation of $\phi_{\mathrm{FM}}$ with temperature can be interpreted by assuming that the non-radiative de-excitation of tryptophan is due to a temperature-independent intersystem crossing with rate constant $k_{1}^{0}$ and a temperature-dependent internal conversion process with rate constant $k_{\mathrm{GM}}=$ $A \exp (-\Delta E / k T)$ with $A$ and $\Delta E$ determined as described above. Values of triplet yields in the same temperature range and spectra for mix tures of solvents of different polarities in various proportions must be obtained to test the validity of this interpretation.

The resolved fluorescence spectra do not show a wavelength shift with ethanol and water solutions at room temperature. Examples of fluorescence spectra measured at different times after excitation by a spark at $280 \mathrm{~nm}$ are

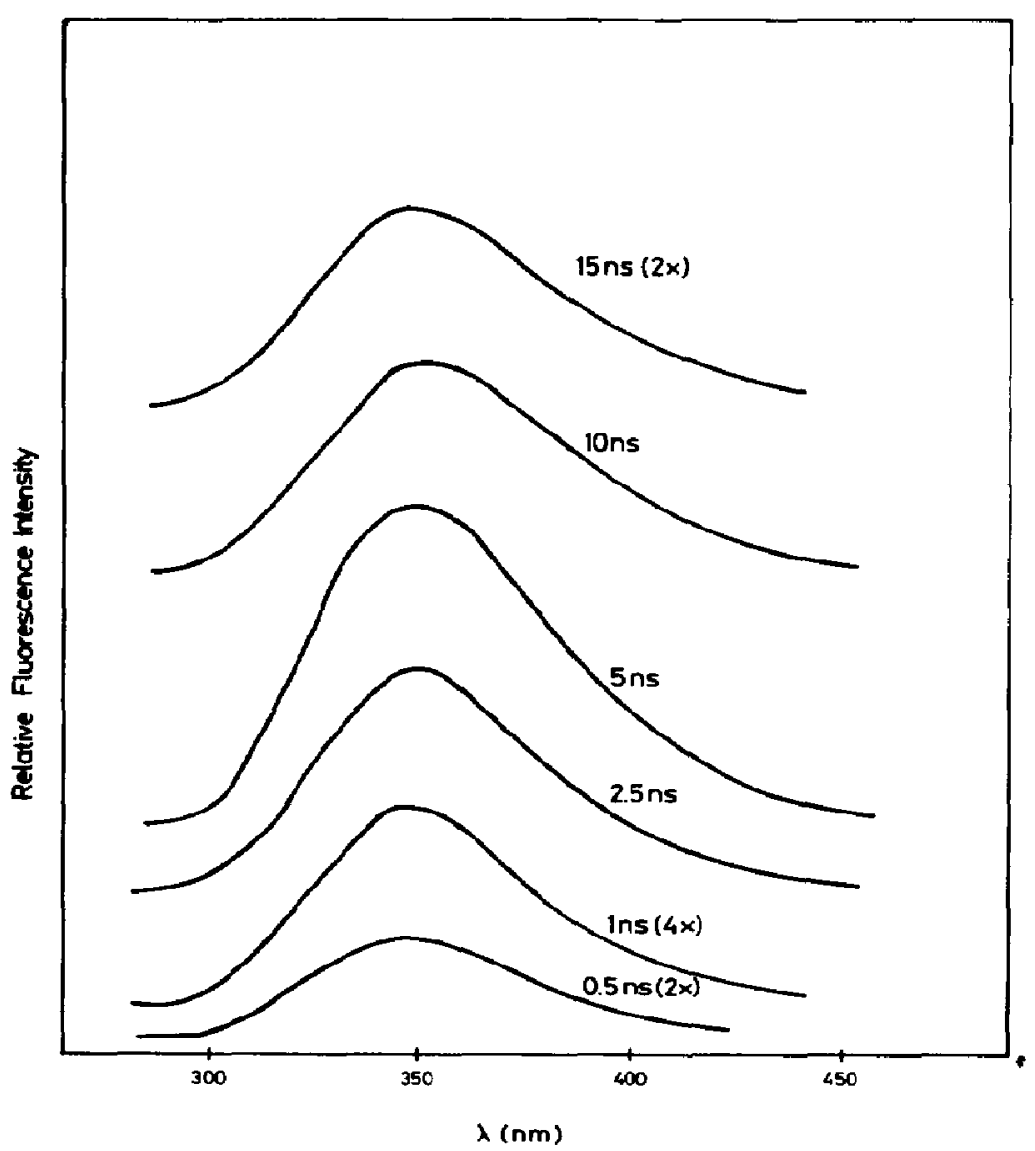

Fig. 4. Time-resolved fluorescence spectra of tryptophan in water at room temperature at different times after pulse initiation (sensitivity in parentheses). 
shown in Fig. 4 for neutral aqueous solutions. The times given on the curves refer to the initial rise of the decay curve and the spectra are obtained without correction. Time-resolved fluorescence spectra for tryptophan in glycerol at $253 \mathrm{~K}$ are shown in Fig. 5. At room temperature only a slight spectral shift is apparent and after $8 \mathrm{~ns} \lambda_{\max }$ is in the same position as obtained in steady state conditions. At lower temperatures a marked shift is found which is dependent on the time elapsed after pulse initiation. For the same time lapse the red shift increases with temperature as is also shown in Fig. 5 .

It is known that in solution tryptophan acts as a zwitterion but this does not seem to explain the anomalous behaviour of the fluorescent state. In fact the absorption spectra do not shift in the same solvents and large Stokes shifts have also been found for indoles in polar solvents $[4,15]$. This has been attributed either to solvent orientation polarization with the excited state dipole [12] or to the formation of an exciplex [16]. Eisinger and Navon [12] have precluded the existence of a solute-solvent exciplex because no iso-emissive points could be found. However, it should be noted that the existence of iso-emissive points is complex when temperature is the variable parameter [17]. The absorption spectra of indole show a large overlap of the ${ }^{1} L_{a}$ and ${ }^{1} L_{b}$ bands [15], and a reversal of ${ }^{1} L_{a}$ and ${ }^{1} L_{b}$ states due to solvent interaction was proposed by Lippert [18] .

The results obtained are tentatively interpreted using the model illustrated in Fig. 6 in which the potential energy is represented as a function of the solvent orientation polarization. In the absorption process both the ${ }^{1} \mathbf{L}_{b}$

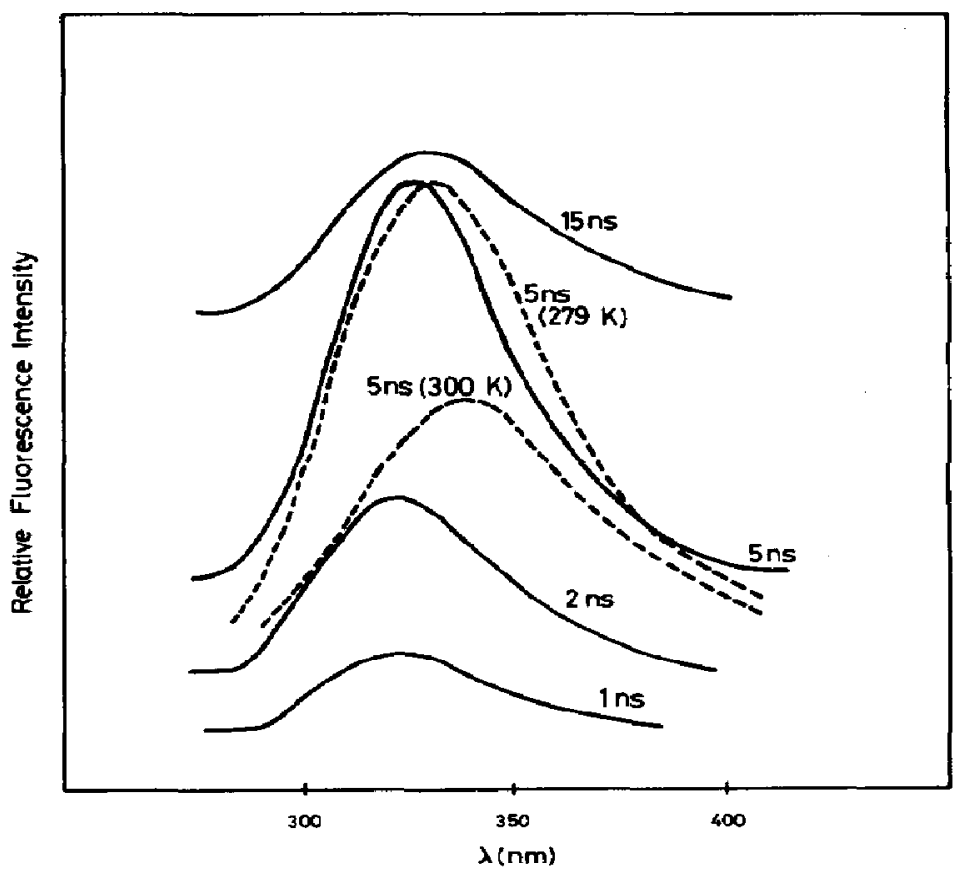

Fig. 5. Time-resolved fluorescence spectra of tryptophan in glycerol: at $253 \mathrm{~K}$;

-.-- at 279 and $300 \mathrm{~K} 5 \mathrm{~ns}$ after pulse initiation. 


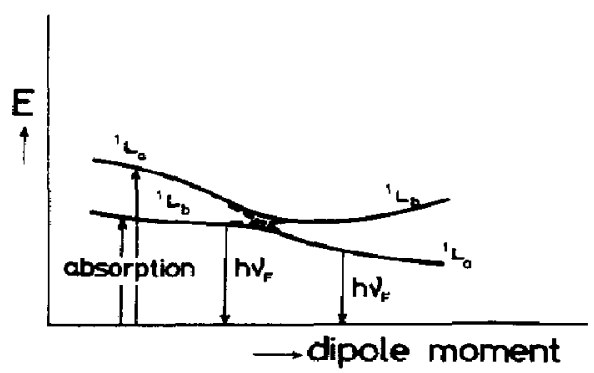

Fig. 6. Energy of ${ }^{1} L_{a}$ and ${ }^{1} L_{b}$ as a function of dipole moment.

and ${ }^{1} \mathrm{~L}_{\mathrm{a}}$ states are populated with a consequent overlap in the absorption spectrum, which is observed for indole solutions [15].

In weak interacting solvents the dipole orientation is small and fluorescence is mainly from ${ }^{1} \mathrm{~L}_{b}$ owing to the rapid vertical relaxation from ${ }^{1} \mathbf{L}_{a}$ to ${ }^{1} L_{b}$. This is the case for fluorescence in ethanol at room temperature $\left(\lambda_{\max }=\right.$ $335 \mathrm{~nm}$ ) and for polycrystalline solid samples in which the medium effect is negligible and the fluorescence is also mainly from ${ }^{1} \mathrm{~L}_{b}\left(\lambda_{\max }=329 \mathrm{~nm}\right)$.

With a more polar environment an inversion of ${ }^{1} \mathbf{L}_{b}$ and ${ }^{1} \mathbf{L}_{a}$ occurs during the lifetime of the excited state if the viscosity is not high enough to prevent solvent orientation. This inversion could result from mixing of the states associated with the avoided crossing produced by solvent perturbation [19]. This correlates with the fact that the dipole moment in going from the ground state to ${ }^{1} L_{k}$ increases in indoles by a factor of 3 but for ${ }^{1} \mathbf{L}_{b}$ is about the same [4]. The results obtained have shown that at low viscosity the interaction with the excited state dipole takes place in less than $1 \mathrm{~ns} . \lambda_{\max }$ is then red shifted as in the case of water and glycerol solutions above room temperature. An increase in the solvent viscosity can prevent the achievement of the correct orientation during the lifetime of the excited state with a consequent spectral blue shift. The reversal due to solvent interaction then takes place in the nanosecond scale and as a result a mixture with various proportions of $a$ and $b$ fluorescence is obtained at intermediate temperatures. At very high viscosities the interaction is totally prevented as in the case of glycerol solutions at $173 \mathrm{~K}\left(\lambda_{\max }=324 \mathrm{~nm}\right)$ in which medium phosphorescence of tryptophan is also observed.

\section{Acknowledgments}

The authors gratefully acknowledge partial financial support from a NATO grant. Thanks are also due to Professor R. B. Cundall of the University of Salford, Gt. Britain, and Dr. M. D. Lumb of the University of Manchester and Institute of Science and Technology, Gt. Britain, for many helpful discussions. 


\section{References}

1 E. A. Burnstein, N. S. Vedenkina and M. N. Inkova, Photochem. Photobiol., 18 (1973) 263 .

2 G. Laustriat and D. Gerard, in J. B. Birks (ed.), Excited States of Biological Molecules, Wiley, London, $1976, \mathbf{p} .388$.

3 R. J. Cherry, FEBS Lett., 55 (1975) 1.

4 Ming Sun and Pill-Soon Sing, Photochem. Photobiol., 25 (1977) 3, and referenceg therein.

5 G. Weber, in J. B. Birks (ed.), Excited States of Biological Molecules, Wiley, London, $1976, p .363$.

6 M. D. Lumb, C. Lloyd Braga and L. C. Pereira, Trans. Faraday Soc., 65 (1969) 1992.

7 J. M. Borges de Almeida, M. D. Lumb and L. C. Pereira, to be published.

8 L. C. Pereira, I. C. Ferreira and M. P. F. Thomaz, Chem. Phys. Lett., 43 (1976) 157. L. C. Pereira, Ph.D. Thesis Univ. Nottingham, 1972.

9 K. D. Mielenz, E. D. Cehelnik and R. L. Mckenzie, J. Chem. Phys., 64 (1976) 370.

10 I. Tatischeff and R. Klein, Photochem. Photobiol., 22 (1975) 221; in J. B. Birks (ed.), Excited States of Biological Molecules, Wiley, London, 1976, p. 375.

11 M. F. Thomaz, I. Barradas and J. A. Ferreira, J. Lumin., 11 (1975) 55.

12 J. Eisinger and G. Navon, J. Chem. Phys., 50 (1969) 2069.

13 F. W. J. Teale and G. Weber, Biochem. J., 65 (1957) 476.

14 R. B. Cundal and L. C. Percira, J. Chem. Soc. Faraday Trans. 2, 68 (1972) 1152.

15 M. S. Walker, T. W. Bednar, R. Lumry and F. Humphries, Photochem. Photobiol., 14 (1971) 147.

16 M. S. Walker, T. W. Bednar and R. Lumry, J. Chem. Phys., 47 (1967) 1020.

17 A. J. H. Al-Wattar and M. D. Lumb, Chem. Phys. Lett., 8 (1971) 331.

18 E. Lippert, Adv. Mol. Spectrosc., 1 (1962) 443; in J. B. Birks (ed.), Organic Molecular Photophysics, Vol, 2, Wiley, London, 1975.

19 J. B. Birks, G. N. R. Tripathi and M. D. Lumb, in publication. 